\title{
Nanoscale Mapping of Interfacial Electrical Transport in Graphene-MoS 2 Heterostructures with STEM-EBIC
}

Edward R. White ${ }^{1,2}$, Alexander Kerelsky², William A. Hubbard ${ }^{2}$, Rohan Dhall ${ }^{3}$, Stephen B. Cronin ${ }^{3}$, Matthew Mecklenburg ${ }^{4}$, and B. C. Regan ${ }^{2}$

1. Department of Chemistry, Imperial College London, London SW7 2AZ, UK.

2. Department of Physics \& Astronomy and California NanoSystems Institute, University of California, Los Angeles, California, 90095, USA.

3. Department of Electrical Engineering, University of Southern California, Los Angeles, California, 90089, USA.

4. Center for Electron Microscopy and Microanalysis, University of Southern California, Los Angeles, California, 90089, USA.

After the discovery of graphene and other two-dimensional materials, the creation of composites formed by stacking individual 2D crystals soon followed [1]. These heterostructures display a variety of extraordinary properties, ranging from itinerant magnetism to superconductivity. Optimal device performance is achieved when the interface between neighboring crystals is free of debris left from the fabrication process. Despite considerable effort to minimize transfer residue, current transfer techniques inevitably leave contamination at the crucial inter-crystal interfaces. Since these interfaces are buried, characterizing them is challenging; current methods are either indirect, or can only demonstrate a correlation of debris with poor device performance. In an extension of previous work [2], here we describe a powerful technique to study the quality of buried interfaces: STEM-EBIC combines local transport measurements with plan-view imaging to reveal the direct impact interfacial nanoscale debris has on device performance.

Graphene- $\mathrm{MoS}_{2}$-graphene (GMG) heterostructures show a strong photo-response and are attractive candidates for flexible photovoltaic devices [3]. A photocurrent map of a GMG heterostructure shows the spatial variation of the photo-generated signal; the map in Figure 1a shows mostly uniform contrast with some micron-sized dark spots away from the lateral boundaries of the constituent materials. An EBIC map of the same device (Figure 1b) shows the same general features, but reveals details not detectable with the diffraction-limited photocurrent measurement. A network of dark patches connected by thin dark lines is apparent, with the patch size ranging from $100 \mathrm{~nm}$ to $2 \mu \mathrm{m}$. In both photocurrent and EBIC measurements a localized probe produces electron-hole pairs that are separated by the internal electric field. Since they probe similar device physics, it is unsurprising that the maps share qualitative similarities. The EBIC map, however, improves on the resolution of the photocurrent map by an order of magnitude [4].

The dark patches indicate near zero induced current, and clearly represent inferior local performance. Comparison of the higher resolution EBIC map (Figure 1c) with the simultaneously acquired ADF STEM image (Figure 1d) reveal that the dark patches coincide with regions of increased ADF intensity. Interfacial debris explains both signal changes. The contamination causes poor local connection at the interface, preventing efficient charge separation and decreasing the EBIC signal. The additional mass scatters more electrons into the ADF detector. Thus STEM-EBIC reveals the direct connection between interfacial contamination and the electrical properties of the device [5]. 
References:

[1] A Geim and I Grigorieva, Nature 499 (2013), p. 419.

[2] E White et al., Microscopy and Microanalysis 20 S3 (2014), p. 172.

[3] L Britnell et al., Science 340 (2013), p. 1311.

[4] E White et al., Applied Physics Letters 107 (2015), p. 223104.

[5] This work has been supported by FAME, one of six centers of STARnet, a Semiconductor Research Corporation program sponsored by MARCO and DARPA, and by National Science Foundation Award No. DMR-1206849. The authors acknowledge the use of instruments at the Electron Imaging Center for NanoMachines supported by NIH 1S10RR23057 and the CNSI at UCLA, and the Zeiss center in Peabody, MA.
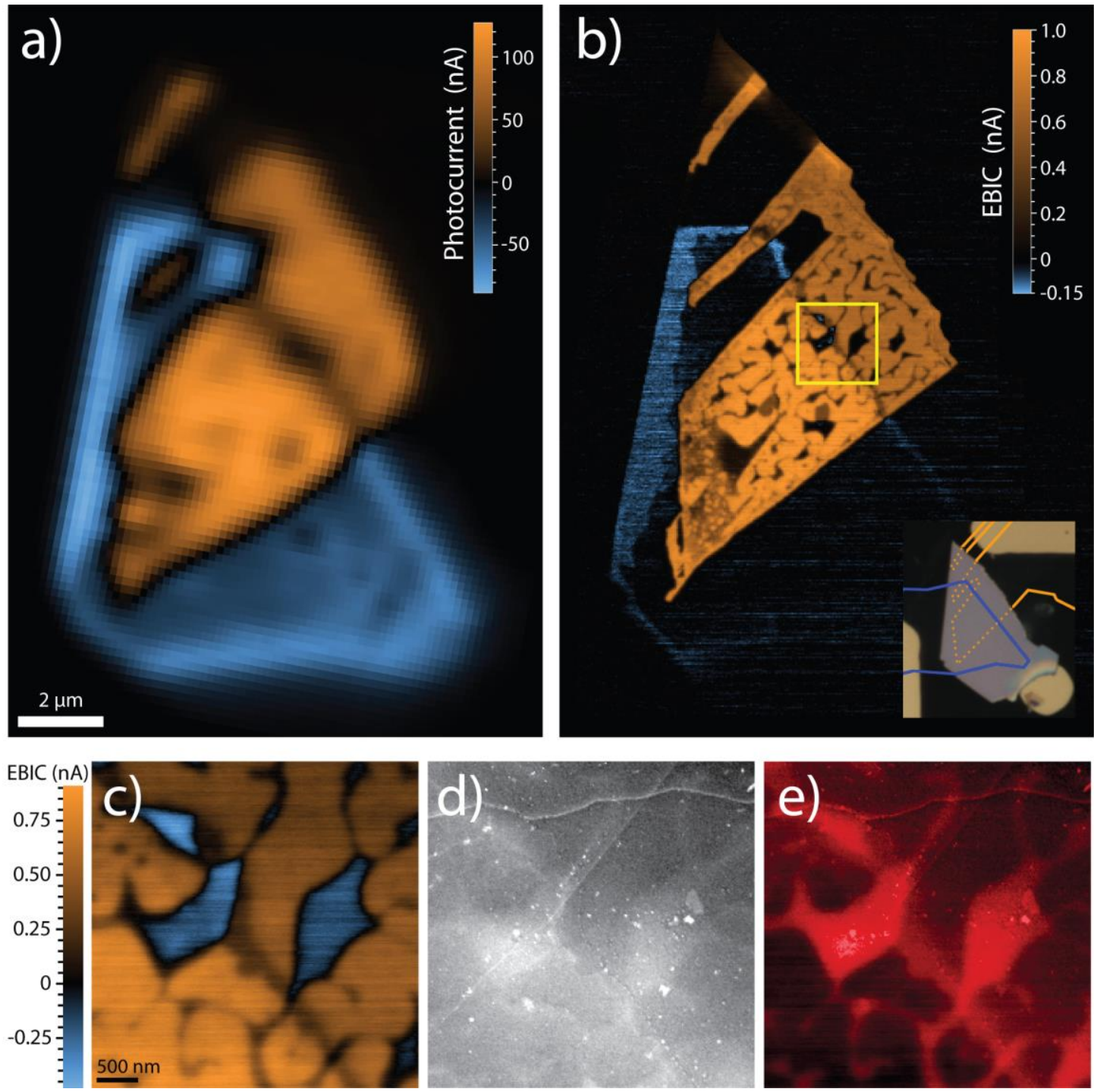

Figure 1. (a) Photocurrent and (b) EBIC maps of a graphene-MoS $\mathrm{S}_{2}$-graphene heterostructure. A photograph of the heterostructure with the top (bottom) graphene electrodes outlined in blue (orange) is shown in the inset. (c) Higher resolution EBIC map of the region outlined by the yellow square. (d) Simultaneously acquired ADF STEM image. (e) Colorized composite of the EBIC map and ADF STEM image. Positive current flows away from the bottom-graphene electrode (orange) and negative current flows away from the top-graphene electrode (blue) in all maps. 